\title{
Velopharyngeal closure analysis using four- dimensional computed tomography: a pilot study of healthy volunteers and adult patients with cleft palate
}

Yoshikazu Kobayashi $i^{*}$ D, Daisuke Kanamori ${ }^{2}$, Naoko Fujii ${ }^{3}$, Yumi Kataoka ${ }^{4}$, Emiko Hirai ${ }^{5}$, Satoshi Yoshioka ${ }^{5}$, Koji Satoh ${ }^{1}$, Hiroshi Toyama ${ }^{6}$, Kensei Naito ${ }^{5}$ and Koichiro Matsuo ${ }^{1}$

\begin{abstract}
Background: Nasopharyngoscopy is a common method to evaluate velopharyngeal closure in patients with cleft palate. However, insertion of a fiberoptic nasopharyngoscope causes discomfort in patients. The aim of this study was to estimate the reliability of short-time exposure images obtained using 320-row area detector computed tomography (320-ADCT) as a novel evaluation method for the assessment of velopharyngeal function.
\end{abstract}

Methods: We evaluated five healthy adult volunteers and five postoperative adult patients with cleft palate. During a 3.3-s imaging exposure, the participants were asked to perform two tasks: nasal inspiration and subsequent oral expiration through a catheter into a water-filled cup. The movement of the velopharyngeal structures was recorded during each examination, and the presence of velopharyngeal insufficiency (VPI) and velopharyngeal closure (VPC) patterns were estimated. If VPI was detected, the cross-sectional area was also calculated. Cohen's kappa and weighted kappa coefficients were used to evaluate the concordance of nasopharyngoscopy and 320-ADCT evaluation.

Results: Speech pathology evaluation did not reveal hypernasality in any study participant. Micro-VPI was detected by nasopharyngoscopy in one healthy volunteer and two patients. 320-ADCT detected micro-VPI in two more patients. The cross-sectional area of the VPI in these subjects ranged from 2.53 to $16.28 \mathrm{~mm}^{2}$. Nasopharyngoscopy and 320ADCT were concordant in detecting VPI in eight participants $(K=0.6)$ and in assessing VPC patterns in nine $(K=0.82)$. Moreover, images obtained using 320-ADCT allowed for reduced dead angle and, thus, easy detection of micro-VPI and Passavant's ridges.

Conclusion: Although the radiation exposure cannot be ignored, our novel evaluation method using 320-ADCT enables more detailed evaluation of VPC than nasopharyngoscopy. Future studies should investigate the relationship between 320-ADCT findings and speech pathology evaluations.

Keywords: Velopharyngeal insufficiency, Nasopharyngoscopy, 320-row area detector computed tomography, Speech pathology, Palatoplasty, Kinematic analysis

\footnotetext{
* Correspondence: y.kobayashi.oms@gmail.com

${ }^{1}$ Department of Dentistry and Oral-Maxillofacial Surgery, Fujita Health

University, School of Medicine, 1-98, Dengakugakubo, Kutsukake, Toyoake,

Aichi 470-1192, Japan

Full list of author information is available at the end of the article
}

(c) The Author(s). 2019 Open Access This article is distributed under the terms of the Creative Commons Attribution 4.0 International License (http://creativecommons.org/licenses/by/4.0/), which permits unrestricted use, distribution, and reproduction in any medium, provided you give appropriate credit to the original author(s) and the source, provide a link to the Creative Commons license, and indicate if changes were made. The Creative Commons Public Domain Dedication waiver (http://creativecommons.org/publicdomain/zero/1.0/) applies to the data made available in this article, unless otherwise stated. 


\section{Background}

Velopharyngeal insufficiency (VPI) is commonly observed in patients with cleft palate due to the structural anatomical abnormality of the soft palate. It can affect both speech and swallowing, which is of major concern in growing children [1]. Multiple surgical or nonsurgical approaches are used to improve velopharyngeal function (VPF), and various examinations are used to assess VPF. Although the evaluation of hypernasality by speech pathology is the gold standard for assessing VPF after cleft repair, an objective VPF analysis is essential for choosing the appropriate surgical procedure for secondary surgery for VPI. In addition to perceptual speech evaluation, the combination of lateral cephalogram and nasopharyngoscopy is commonly used for assessing the anatomical abnormalities causing VPI. However, lateral cephalogram only evaluates the two-dimensional (2D) elevation or thickness of the soft palate. Although nasopharyngoscopy is useful for assessing the anatomical abnormality, it can cause discomfort to the patient, due to the insertion of the fiberoptic nasopharyngoscope into the nasopharyngeal space, which increases the difficulty of the examination. Moreover, multiple examinations are time-consuming.

In 2008, the 320-row area detector computed tomography (320-ADCT) scanner first appeared as a medical imaging apparatus that could append the time phase to three-dimensional (3D) images, obtaining so-called "four-dimensional (4D) images [2, 3]". This equipment loads a broad area detector $(0.5 \mathrm{~mm} \times 320$ rows $=160$ $\mathrm{mm})$ and a cone-beam irradiation source onto a highspeed rotating gantry. It can thus provide wide-range 3D images without moving the patient's bed and can generate $4 \mathrm{D}$ images of the same position continuously or intermittently. Therefore, it is of great significance in the evaluation of cardiovascular disease [3], dysphagia [4, 5], and cerebrovascular disease $[6,7]$.

In 2015, Sakamoto et al. first reported the clinical application of 320-ADCT to estimate VPF in patients with cleft palate [8]. The authors evaluated five children (aged 4-10 years) with persistent VPI postpalatoplasty with 10-s imaging, during vowel phonation and swallowing. They concluded that 320ADCT provides detailed morphological and kinematic analysis of VPC and may offer advantages over conventional procedures [8]. However, the authors also advised that the high exposure dose during imaging $(4.44 \pm 1.64 \mathrm{mSv})$ could not be ignored in pediatric patients.

The aim of this pilot study was to estimate the reliability of short-time and dose-reduced exposure imaging by 320-ADCT as a novel evaluation method for the assessment of VPC before use in pediatric patients.

\section{Methods}

\section{Subjects}

We evaluated five healthy volunteers (two men and three women, age range 27 to 31 years) and five postoperative patients with cleft palate (four men and one woman, age range 20 to 24 years). All patients had undergone two-stage palatoplasty without pharyngeal flap operation.

The study was conducted in accordance with the Declaration of Helsinki (1964) and was approved by the Fujita Health University Ethics Review Committee (reference number: HM17-038). All participants were sufficiently informed about the purpose of this study, including the fact that it did not involve therapeutic, but exploratory research, and provided written informed consent. The participants were also informed of the estimated radiation exposure dose by 320-ADCT (about $1.65 \mathrm{mSv}$ ), as cited in earlier research [9].

\section{Procedure}

Speech samples, including single sounds, syllable repetition, and connected speech, were corrected for speech pathology evaluation. Three well-trained speech-languagehearing therapists assessed the existence of hypernasality based on these samples.

320-ADCT (Aquilion ONE ${ }^{\text {tw }} /$ Genesis Edition. Toshiba Medical Systems Corp., Tochigi, Japan) and nasopharyngoscopy were also performed for all the participants. Participants were asked to perform two successive tasks: nasal inspiration and subsequent oral expiration through a catheter into a water-filled cup.

During the CT scan, each subject lay down horizontally on the bed and performed the task, following a signal given by an examiner standing beside the CT scanner. Scanning was started immediately after the onset of inspiration. Expiration was continued $1 \mathrm{sec}$ after the start and until the end of the scanning. We used a 14-French gauge (4.7- $\mathrm{mm}$ outer diameter, $47-\mathrm{cm}$ long) catheter (Nurvie ${ }^{\mathrm{rm}}$, Covidien Japan, Tokyo) and disposable clear PET cups $(370 \mathrm{~mL})$ half-filled with water (Fig. 1). This ensured that the examiner could easily ascertain that the task was being performed, by the formation of bubbles in the water.

The scanning parameters were set as follows: field of view, $160 \mathrm{~mm}$; tube voltage, $120 \mathrm{kV}$; tube current, $60 \mathrm{~mA}$; and exposure time, $3.30 \mathrm{~s}(0.275 \mathrm{~s} /$ rotation $\times 12$ rotations $)$. To improve the temporal resolution, we implemented a half-reconstruction technique by which images were generated from 0.138-s data for image generation. The images were drawn with $0.5-\mathrm{mm}$ slice thickness and transferred to a medical imaging workstation (Ziostation2, Ziosoft, Inc., Tokyo, Japan) where 4D images of airway mobility (airway-mode), 4D images in virtual endoscopy mode, and 


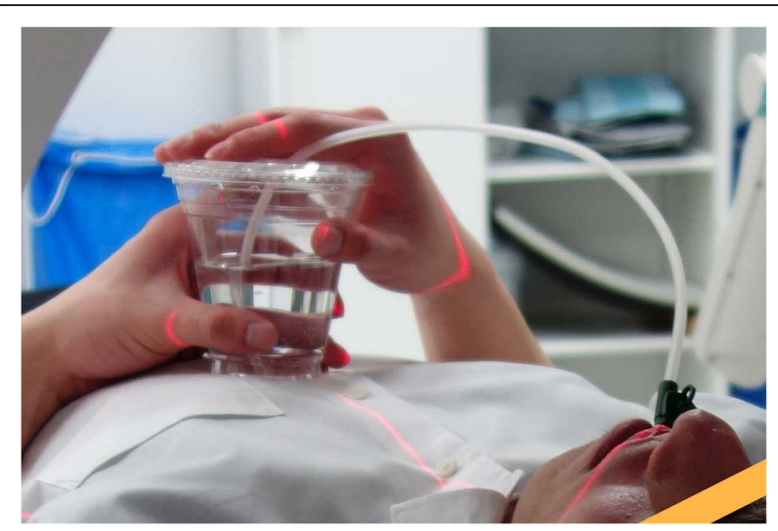

Fig. 1 A participant performing the study's tasks (Authors' original picture). Each participant performed two tasks: nasal inspiration and subsequent forceful expiration through a catheter into a water-filled cup

multiplanar reconstruction (MPR) images were generated (Fig. 2). Airway-mode, virtual endoscopy-mode, and MPR images had a window length of 500, 75, and $40 \mathrm{HU}$, and a window width of 500, 500, and $400 \mathrm{HU}$, respectively.

Nasopharyngoscopy was performed by two welltrained otorhinolaryngologists. During the examination, subjects lay down on the bed in the same position used during the CT procedure.

The movement of the velopharyngeal structures was recorded during each examination (Additional file 1: Video S1), and the presence of VPI and VPC patterns were estimated. VPC patterns were categorized into four groups: coronal, sagittal, circular, and circular with Passavant's ridge, in accordance with earlier reports [10] (Fig. 3). The minimum cross-sectional area of the nasopharynx was measured parallel to the palatal plane, using MPR images obtained during blowing. The volume of the CT dose index $\left(\mathrm{CTDI}_{\mathrm{vol}}\right)$ and dose length product (DLP) for each scan were around $16.10 \mathrm{mGy}$ and $258.80 \mathrm{mGy} \cdot \mathrm{cm}$, respectively.

\section{Statistical analysis}

We assessed the consistency of the results obtained by $\mathrm{CT}$ and nasopharyngoscopy in detecting the presence of VPI, as the primary outcome measure, using Cohen's kappa coefficient. Moreover, the consistency of the two methods for detecting VPC patterns was evaluated using the weighted kappa coefficient. We also evaluated consistency of these two methods, with respect to VPI and hypernasality. Moreover, we assessed the timing of the movements of the soft palate, lateral pharyngeal wall (LPW), posterior pharyngeal wall (PPW), and tongue, to evaluate the motor coordination of the anatomical structures associated with the VPC.
Statistical analysis was conducted using $\mathrm{JMP}^{\circ}$ v. 13.2 (SAS Institute Inc., Cary, NC, USA), and statistical significance was accepted when $p<0.05$.

\section{Results}

Micro-VPI was detected by nasopharyngoscopy in one healthy volunteer and in two patients with cleft palate (Table 1).

320-ADCT detected micro-VPI in two more patients. The cross-sectional area of VPI in these five participants ranged from $2.53-16.28 \mathrm{~mm}^{2}$. Nasopharyngoscopy and 320 -ADCT were concordant in their assessment in $8 / 10$ participants $(\kappa=0.6, p=0.04)$, and in assessing the VPC patterns in $9 / 10$ participants $(\kappa=0.82, p=0.0009)$. Hypernasality was not observed in any of the cases, even though, some subjects exhibited the presence of VPI. Passavant's ridges were seen in four postoperative patients. The motor coordination of the anatomical structures associated with VPC for each subject is indicated in Fig. 4.

\section{Discussion}

In this study, we estimated the reliability of a novel evaluation method, "virtual nasopharyngoscopy," for the assessment of VPC, using short-time exposure 320ADCT.

Although nasopharyngoscopy provides useful 3D information, allowing for the detection of the region and the severity of VPI, it causes pain and distress to younger children or patients, as it induces a vomiting reflex. Thus, in such cases, surgical intervention for VPI, performed without presurgical nasopharyngoscopy, must rely on the empirical assumptions of skilled surgeons. Moreover, incomplete visualization due to the dead angle created by the elevated soft palate is an obstacle during evaluation [11]. Several studies have estimated VPI or VPF using dynamic magnetic resonance imaging. Although it is a non-invasive technique, which can be repeated without the risk of radiation exposure, its low imaging speed, narrow spatial coverage, and noise may interfere in the examination of pediatric patients, when compared to CT $[12,13]$.

The first assessment of VPF with 320-ADCT in patients with cleft palate was reported in 2015 by Sakamoto et al. [8]. In that study, the authors evaluated five children with persistent VPI, following palatoplasty, using three tasks during a $\sim 10$-s CT exposure: sustained production of the vowels [/a:/] and [/i:/], and swallowing. They concluded that $4 \mathrm{D}-\mathrm{CT}$ produces clear and detailed images with lesser stress and pain, shorter examination duration than other imaging modalities, and quantitative VPC evaluation [8]. However, they reported that the radiation exposure of approximately $4 \mathrm{mSv}$, which is larger than that required in cephalometry, videofluoroscopy, 


\section{A}

\section{D airway-mode}

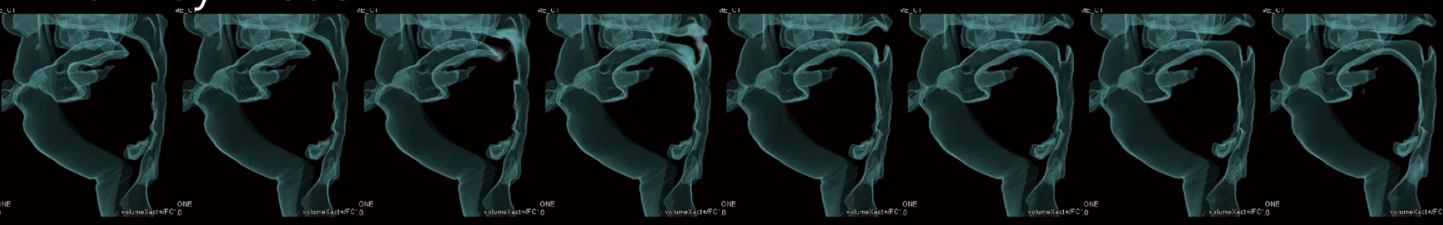

Nasopharyngoscopy

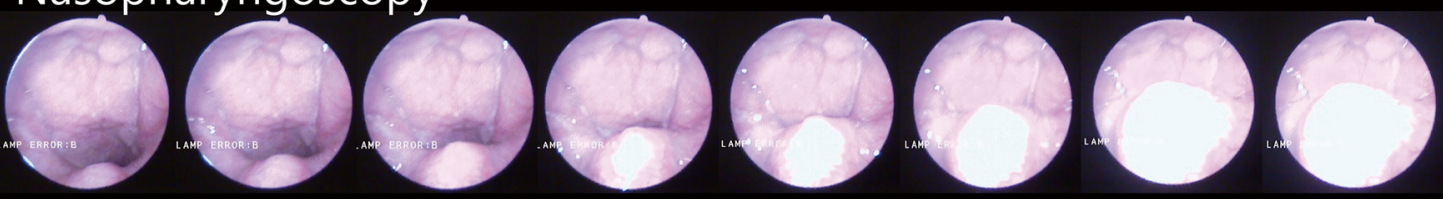

4D virtual nasopharyngoscopy-mode
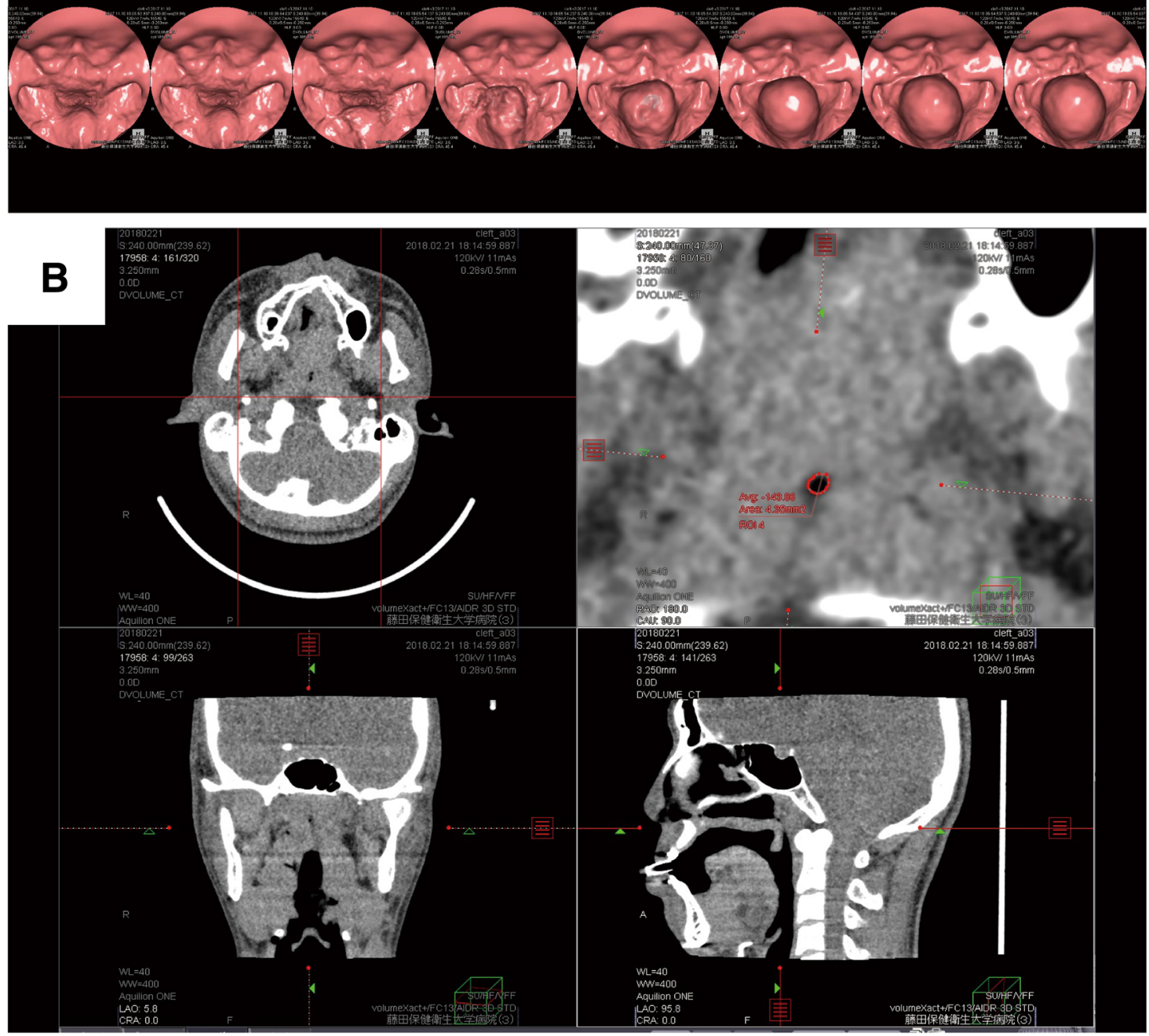

Fig. 2 Computed tomography and nasopharyngoscopy images. a Images of the airway in 4D-mode (top), actual images of nasopharyngoscopy (middle), and 4D images of virtual nasopharyngoscopy-mode (bottom) b Multiplanar reconstruction images to calculate the cross-sectional area of velopharyngeal insufficiency 

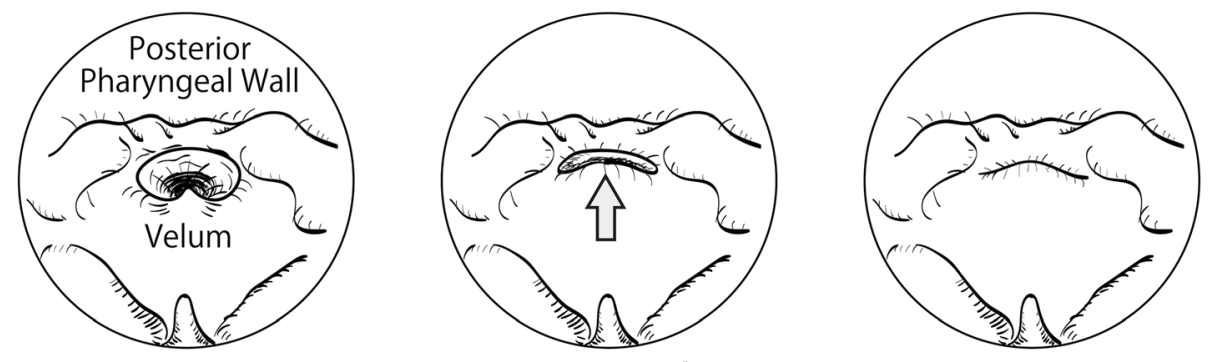

Coronal
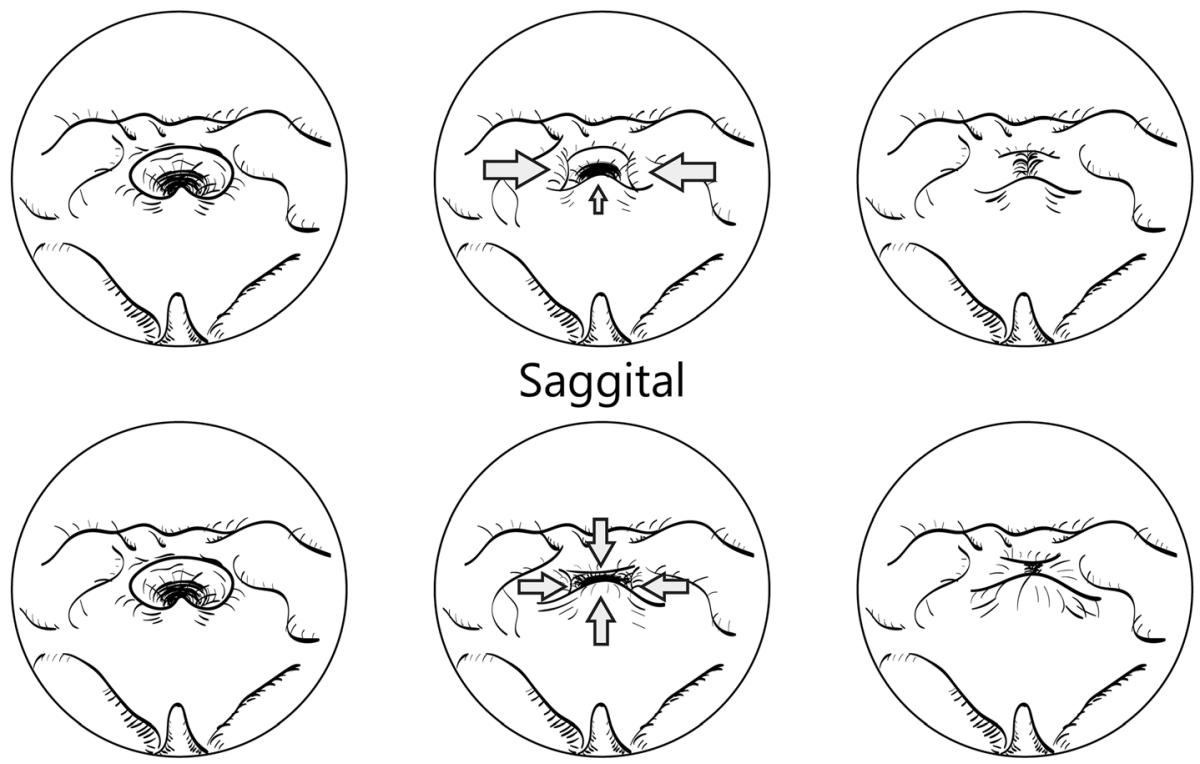

Saggital
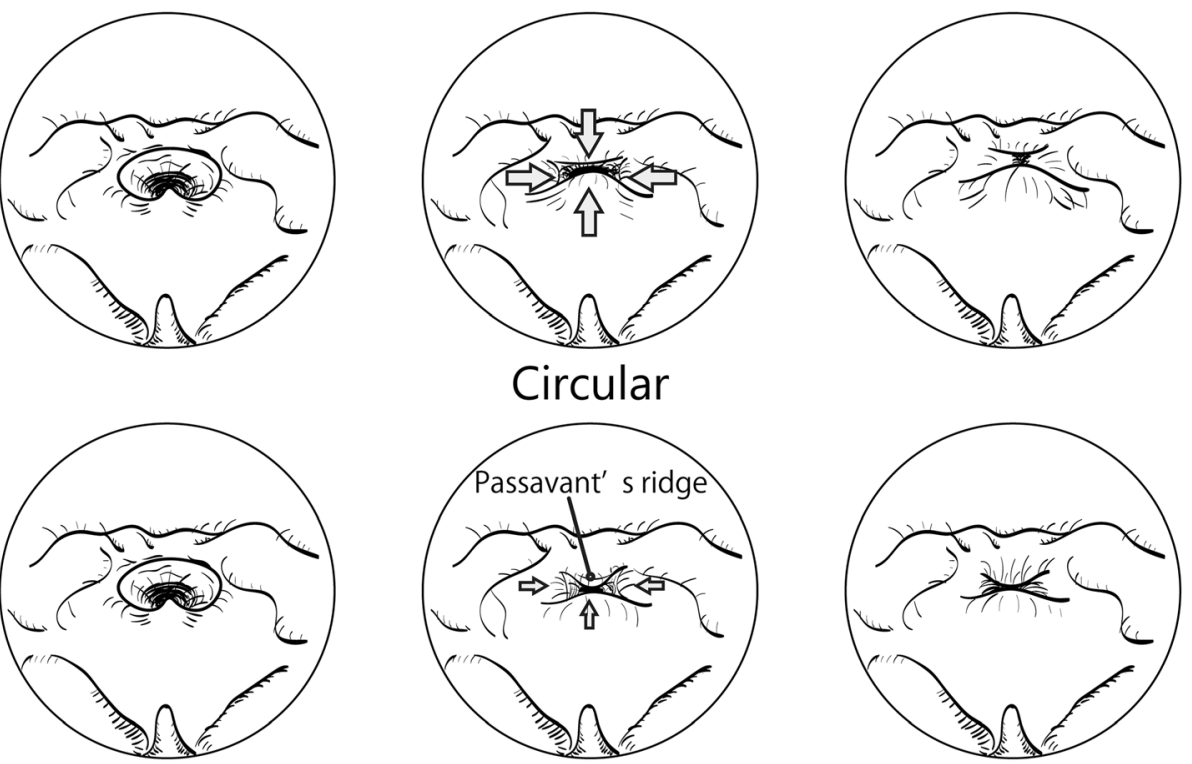

\section{Circular}

\section{Circular with Passavant' s ridge}

Fig. 3 Patterns of velopharyngeal closure. The patterns of velopharyngeal closure are categorized into four groups in accordance with previous literature [10]

and conventional CT [8], was a major drawback of this procedure. In our study, we modified the 320-ADCT scan protocol to shorten the exposure time during VPF assessment. Thus, the existence of VPI and VPC patterns was precisely assessed, consistent with the findings of nasopharyngoscopy. Furthermore, we implemented a quantitative calculation of the nasopharyngeal crosssectional area and dynamically analyzed the anatomical structures associated with VPC, which was not possible earlier, with the sole use of an existing modality. Although none of the subjects in our study exhibited hypernasality during speech evaluation, we detected micro VPI in five out of ten participants, which was not detected by nasopharyngoscopy in two of these participants.

The International Commission on Radiological Protection and several other international organizations have cooperated to find ways to minimize radiation 
Table 1 Distribution of velopharyngeal insufficiency and patterns of velopharyngeal closure

\begin{tabular}{|c|c|c|c|c|c|c|c|}
\hline \multirow[b]{2}{*}{ No. } & \multirow[b]{2}{*}{ age } & \multirow[b]{2}{*}{ sex } & \multicolumn{2}{|c|}{ Nasopharyngoscopy } & \multicolumn{3}{|c|}{ CT evaluation } \\
\hline & & & $\overline{V P I}$ & VPC pattern & $\overline{V P I}$ & VPC pattern & VPI cross-sectional area $\left(\mathrm{mm}^{2}\right)$ \\
\hline Volunteer \#1 & 31 & M & + & circular & + & circular & 3.09 \\
\hline Volunteer \#2 & 31 & $\mathrm{~F}$ & - & circular & - & circular & 0 \\
\hline Volunteer \#3 & 29 & M & - & coronal & - & coronal & 0 \\
\hline Volunteer \#4 & 27 & $\mathrm{~F}$ & - & circular & - & circular & 0 \\
\hline Volunteer \#5 & 30 & $\mathrm{~F}$ & - & circular & - & circular & 0 \\
\hline Adult patient \#1 & 24 & M & - & circular & + & circular with Passavant's ridge & 6.22 \\
\hline Adult patient \#2 & 21 & M & + & circular & + & circular & 16.28 \\
\hline Adult patient \#3 & 22 & M & + & circular with Passavant's ridge & + & circular with Passavant's ridge & 4.35 \\
\hline Adult patient \#4 & 20 & M & - & circular with Passavant's ridge & - & circular with Passavant's ridge & 0 \\
\hline Adult patient \#5 & 21 & $\mathrm{~F}$ & - & circular with Passavant's ridge & + & circular with Passavant's ridge & 2.53 \\
\hline
\end{tabular}

CT computed tomography, VPI velopharyngeal insufficiency, VPC velopharyngeal closure; +, present; -, absent

exposure. Diagnostic reference levels are important methods for optimizing protection, including the computed tomography dose index $\left(\mathrm{CTDI}_{\mathrm{vol}}\right)$ and dose length product (DLP). The $\mathrm{CTDI}_{\mathrm{vol}}$ is a dose index that evaluates the performance of CT scanners, while the DLP is the product of CTDI $\mathrm{I}_{\mathrm{vol}}$ and scan length that estimates the total amount of radiation [14]. In 2015, the Japan Network for Research and Information on Medical Exposure published the report "Diagnostic Reference Levels Based on Latest Surveys in Japan." [15] In this report, the CTDI $\mathrm{vol}_{\mathrm{vol}}$ for head and chest CT for children aged 6-10 years was set at 60 and $15 \mathrm{mGy}$, respectively, while the corresponding DLP was set at 850 and $410 \mathrm{mGy} \cdot \mathrm{cm}$. In our study, the values of CTDI $\mathrm{vol}_{\mathrm{vo}}$ and DLP were $16.10 \mathrm{mGy}$ and $258.80 \mathrm{mGy} \cdot \mathrm{cm}$, respectively. Thus, reducing the exposure dose is of the outmost importance when this method is used in pediatric patients.

In this study, all the participants could perform the requisite tasks during the $\mathrm{CT}$ examination after a few rehearsals, except for the first participant, a healthy volunteer, who missed the examiner's sign. This dynamic analysis allowed for some extent of timing errors. This is the most important advantage of this method, as it can be useful during the functional examination of pediatric patients, who have difficulties in performing tasks depending on the examiners' signs.

Moreover, we demonstrated that 4D-CT provides kinematic analysis of the individual anatomical structures associated with VPC. To the best of our knowledge, there are no reports of this time-based analysis in the literature. Based on this analysis, we found that the velum starts to move before the other VPC structures, always, regardless of the presence of VPI or VPC patterns. Future research should address whether these kinematic features affect or cause hypernasality.

This study has some limitations. First, the number of feasible tasks that could be performed during this short-duration of CT scanning was very limited; therefore, some discrepancies might exist between the results obtained from this method and the actual hypernasality. In fact, in this study, we detected micro-VPI in five participants, although none of the participants exhibited hypernasality. The presence or absence of the influence of micro-VPI on hypernasality is interesting, which may be elucidated quantitatively in future studies. Second, the exposure dose used in this method remains higher than the dose used in conventional radiological assessments, such as cephalometry or videofluoroscopy. However, we believe that the exposure time or scanning range could be further improved. Nevertheless, the data obtained using this method are acceptable in selected situations, as in cases of children who cannot receive nasopharyngoscopy or to estimate the size of the flap in those who need to undergo pharyngeal flap surgery. Of course, the largest advantages of CT evaluation when compared to non-irradiating methods are reproducibility, standardization, and a quantitative analysis. We believe that further research will make the best use of these merits and improve the quality of treatment and management in cleft repair cases.

\section{Conclusion}

Nasopharyngoscopy and 320-ADCT have a high concordance rate for evaluating VPI, as well as VPC patterns. Images obtained using 320-ADCT exhibit reduced dead angle, allowing for easy detection of micro-VPI and Passavant's ridge. Although some extent of radiation exposure 

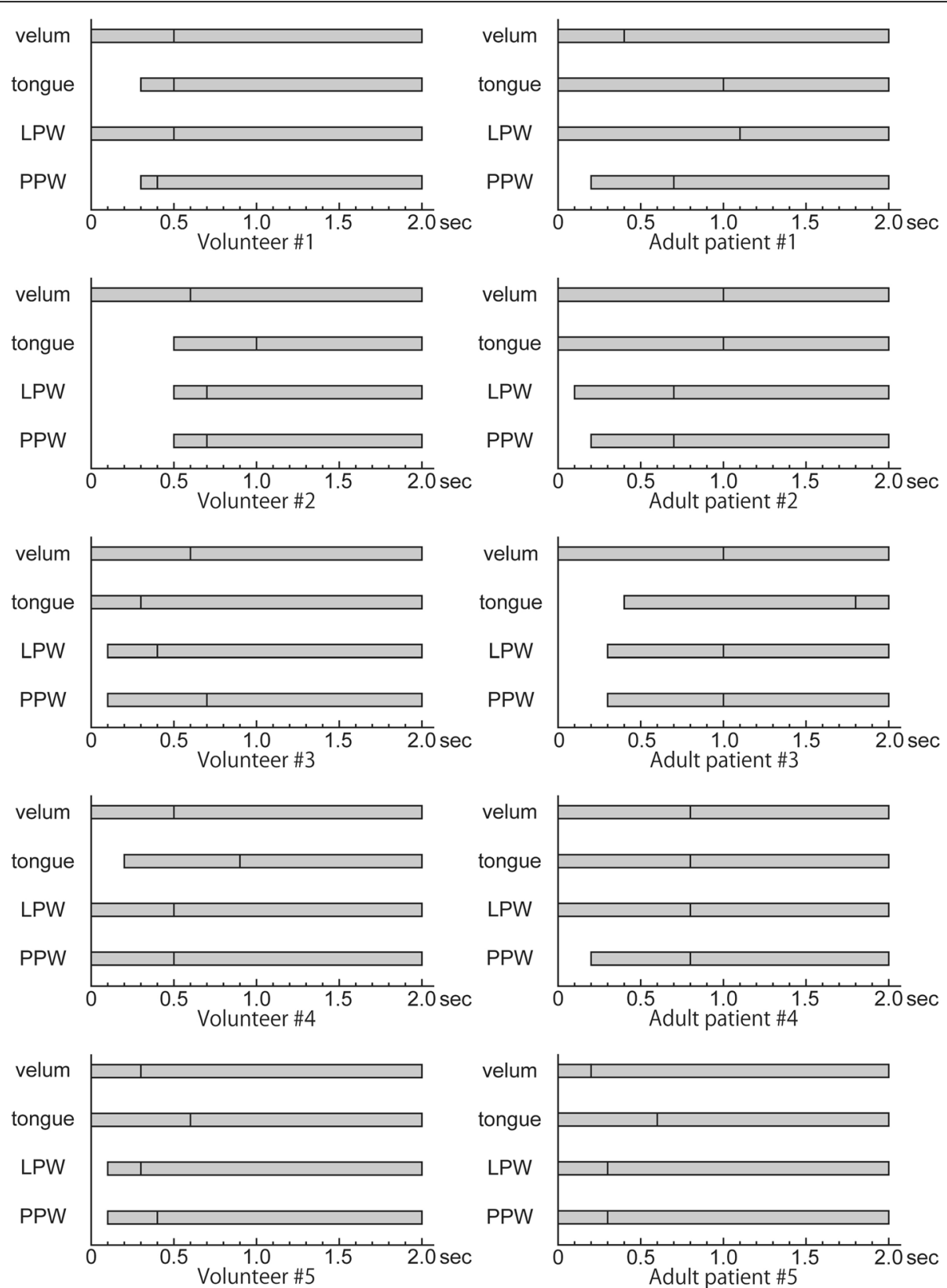

Fig. 4 Motor coordination of anatomical structures associated with velopharyngeal closure in each participant. Each crossbar indicates the continuity of motion. The vertical line in each crossbar indicates the onset of motion. LPW, lateral pharyngeal wall; PPW, post pharyngeal wall

cannot be ignored, our novel evaluation method using 320-ADCT allows for a more detailed evaluation of VPC than nasopharyngoscopy. Future studies should investigate the relationship between findings from 320-ADCT images and those from speech pathology evaluations.

\section{Additional file}

Additional file 1: Video S1. The actual footage of 4D-CT obtained from volunteer \#3. (WMV $2806 \mathrm{~kb}$ )

\section{Abbreviations}

2D: Two-dimensional; 320-ADCT: 320-row area detector computed tomography; 3D: Three-dimensional; 4D: Four-dimensional; CTDI vol: Volume of the $C T$ dose index; DLP: Dose length product; LPW: Lateral pharyngeal wall; MPR: Multiplanar reconstruction; PPW: Posterior pharyngeal wall; VPC: Velopharyngeal closure; VPF: Velopharyngeal function;

VPI: Velopharyngeal insufficiency

\section{Acknowledgments}

The authors would like to thank Ms. Mari Okuni, Ms. Shoko Fujita, and Ms. Ai Fukushima (speech-language pathologists) for the speech pathology evaluation. 


\section{Authors' contributions}

YKB designed the study and wrote the initial draft of the manuscript. DK, NF, $\mathrm{YKO}, \mathrm{EH}, \mathrm{SY}$, and KS contributed to the design of the study and the analysis and interpretation of data and assisted in the preparation of the manuscript. $\mathrm{HT}, \mathrm{KN}$, and KM contributed to data collection and interpretation, and critically reviewed the manuscript. All authors approved the final version of the manuscript and have agreed to be accountable for all aspects of the work in ensuring that questions related to the accuracy or integrity of any part of the work are appropriately investigated and resolved.

\section{Funding}

This work was supported by JSPS KAKENHI grant number JP17K17299. The funding sponsor had no role in the collection, analysis, or interpretation of the data or in the decision to submit the manuscript for publication.

\section{Availability of data and materials}

The datasets supporting the conclusions of this article are available from the corresponding author on reasonable request.

\section{Ethics approval and consent to participate}

The study was conducted in accordance with the Helsinki Declaration of 1964 and approved by Fujita Health University Ethics Review Committee (reference number: HM17-038). Written informed consent was obtained from all participants.

\section{Consent for publication}

Not applicable.

\section{Competing interests}

The authors declare that they have no competing interests.

\section{Author details}

'Department of Dentistry and Oral-Maxillofacial Surgery, Fujita Health University, School of Medicine, 1-98, Dengakugakubo, Kutsukake, Toyoake, Aichi 470-1192, Japan. ${ }^{2}$ Department of Dentistry, Nanakuri Memorial Hospital, Fujita Health University, 424-1, Oodoricho, Tsu, Mie 514-1295, Japan. ${ }^{3}$ Department of Radiology, Bantane Hospital, Fujita Health University, 3-6-10, Otobashi, Nakagawa-ku, Nagoya, Aichi 454-8509, Japan. ${ }^{4}$ Department of Radiology, Fujita Health University Hospital, 1-98, Dengakugakubo, Kutsukake, Toyoake, Aichi 470-1192, Japan. ${ }^{5}$ Department of Otolaryngology, Fujita Health University, School of Medicine, 1-98, Dengakugakubo, Kutsukake, Toyoake, Aichi 470-1192, Japan. ${ }^{6}$ Department of Radiology, Fujita Health University, School of Medicine, 1-98, Dengakugakubo, Kutsukake, Toyoake, Aichi 470-1192, Japan.

Received: 18 April 2019 Accepted: 12 June 2019

Published online: 08 July 2019

\section{References}

1. Rajan S, Kurien M, Gupta AK, Mathews SS, Albert RR, Tychicus D. Velopharyngeal incompetence in patients with cleft palate, flexible video pharyngoscopy and perceptual speech assessment: a correlational pilot study. J Laryngol Otol. 2014;128(11):986-90.

2. Kobayashi Y, Satoh K, Kanamori D, Mizutani H, Fujii N, Aizawa T, Toyama H, Yamada $\mathrm{H}$. Evaluating the exposure dose of 320-row area detector computed tomography and its reliability in the measurement of bone defect in alveolar cleft. J Oral Maxillofac Surg Med Pathol. 2017;29(4):350-7.

3. Rybicki FJ, Otero HJ, Steigner ML, Vorobiof G, Nallamshetty L, Mitsouras D, Ersoy H, Mather RT, Judy PF, Cai T, Coyner K, Schultz K, Whitmore AG, Di Carli MF. Initial evaluation of coronary images from 320-detector row computed tomography. Int J Cardiovasc Imaging. 2008;24(5):535-46

4. Fujii N, Inamoto Y, Saitoh E, Baba M, Okada S, Yoshioka S, Nakai T, Ida Y, Katada K, Palmer JB. Evaluation of swallowing using 320-detector-row multislice CT. Part I: single- and multiphase volume scanning for threedimensional morphological and kinematic analysis. Dysphagi. 2011;26(2): 99-107

5. Inamoto Y, Fujii N, Saitoh E, Baba M, Okada S, Katada K, Ozeki Y, Kanamori D, Palmer JB. Evaluation of swallowing using 320-detector-row multislice CT. Part II: kinematic analysis of laryngeal closure during normal swallowing. Dysphagia. 2011;26(3):209-17.
6. Hayakawa M, Tanaka T, Sadato A, Adachi K, Ito K, Hattori N, Omi T, Oheda M, Katada K, Murayama K, Kato Y, Hirose Y. Detection of pulsation in unruptured cerebral aneurysms by ECG-gated 3D-CT angiography (4D-CTA) with 320-row area detector CT (ADCT) and follow-up evaluation results: assessment based on heart rate at the time of scanning. Clin Neuroradiol. 2014;24(2):145-50

7. Klingebiel R, Siebert E, Diekmann S, Wiener E, Masuhr F, Wagner M, Bauknecht HC, Dewey M, Bohner G. 4-D imaging in cerebrovascular disorders by using 320-slice CT: feasibility and preliminary clinical experience. Acad Radiol. 2009;16(2):123-9.

8. Sakamoto Y, Soga S, Jinzaki M, Yamada Y, Ogata H, Kishi K. Evaluation of velopharyngeal closure by 4D imaging using 320-detector-row computed tomography. J Plast Reconstr Aesthet Surg. 2015;68(4):479-84.

9. Kanamori D, Kagaya H, Fujii N, Inamoto Y, Nakayama E, Suzuki S, Mizutani H, Okada S, Katada K, Saitoh E. Examination of the distance measurement error and exposed dose when using a 320-row area detector CT: a comparison with videofluoroscopic examination of swallowing. Jpn J Compr Rehabil Sci. 2012;2(2011):18-23.

10. Croft CB, Shprintzen RJ, Rakoff SJ. Patterns of velopharyngeal valving in normal and cleft palate subjects: a multi-view videofluoroscopic and nasendoscopic study. Laryngoscope. 1981;91(2):265-71.

11. Honjo I, Mitoma T, Ushiro K, Kawano M. Evaluation of velopharyngeal closure by CT scan and endoscopy. Plast Reconstr Surg. 1984;74(5):620-7.

12. Perry JL, Kuehn DP, Sutton BP, Fang X. Velopharyngeal structural and functional assessment of speech in young children using dynamic magnetic resonance imaging. Cleft Palate Craniofac J. 2017:54(4):408-22.

13. Perry JL, Mason K, Sutton BP, Kuehn DP. Can dynamic MRI be used to accurately identify velopharyngeal closure patterns? Cleft Palate Craniofac J. 2018;55(4):499-507.

14. Valentin J. Managing patient dose in multi-detector computed tomography (MDCT). ICRP publication 102. Ann ICRP. 2007;37(1):1-79 iii.

15. Diagnostic Reference Levels Based on Latest Surveys in Japan -Japan DRLs 2015-. 2015. http://www.radher.jp/J-RIME/report/DRLhoukokusyoEng.pdf. Accessed 4 Dec 2018.

\section{Publisher's Note}

Springer Nature remains neutral with regard to jurisdictional claims in published maps and institutional affiliations.

Ready to submit your research? Choose BMC and benefit from:

- fast, convenient online submission

- thorough peer review by experienced researchers in your field

- rapid publication on acceptance

- support for research data, including large and complex data types

- gold Open Access which fosters wider collaboration and increased citations

- maximum visibility for your research: over $100 \mathrm{M}$ website views per year

At BMC, research is always in progress.

Learn more biomedcentral.com/submissions 